\title{
Effect of Three Protocols of Warming up Involving Stretching on the Kinematic Performance of Horizontal Jumping, Agility and Speed for Soccer Players
}

\author{
Mohamed Abdelsattar Abdelkader ${ }^{1}$, Wadee Mohamed Elmorsi ${ }^{2}$ \\ ${ }^{1}$ Department of Sports Training, Faculty of Physical Education, Mansoura University, Mansoura, Egypt \\ ${ }^{2}$ Department of Sport Kinesiology, Faculty of Physical Education, Mansoura University, Mansoura, Egypt \\ Email address: \\ Dr_mabdalsatar@yahoo.com (M. A. Abdelkader), Dr_mabdalsatar@mans.edu.eg (M. A. Abdelkader), \\ Dr_wadeea@mans.edu.eg (W. M. Elmorsi),Dr_wadeea2005@yahoo.com (W. M. Elmorsi)
}

\section{To cite this article:}

Mohamed Abdelsattar Abdelkader, Wadee Mohamed Elmorsi. Effect of Three Protocols of Warming up Involving Stretching on the Kinematic Performance of Horizontal Jumping, Agility and Speed for Soccer Players. American Journal of Sports Science. Vol. 4, No. 3, 2016, pp. 55-60. doi: 10.11648/j.ajss.20160403.12

Received: April 6, 2016; Accepted: April 15, 2016; Published: April 26, 2016

\begin{abstract}
The aim of this study is to investigate the effect of three different protocols of stretching on the kinematic performance of horizontal jumping, Agility and speed for soccer players. The first protocol is doing ballistic stretching then static stretching exercises (BS-SS). The second protocol is doing static stretching then ballistic stretching exercises (SS-BS). The third protocol is doing soccer passing drills (SPD) for 10 minutes without stretching. The three protocols were applied after 5 minutes of running activities. Dependent variables included a horizontal jump, agility run, and $30-\mathrm{m}$ sprint tests. Also speed, acceleration, and steps frequency were analyzed. This study was applied on 23 trained male student-athletes aged $(20+$ 0.4 years). The (SS-BS) condition $(2.247+0.096 \mathrm{~m})$ showed significant differences $(\mathrm{p}<0.01)$ for longer distance than the (BS-SS) condition $(2.165+0.124)$ and also than the (SPD) condition $(2.197+0.110 \mathrm{~m})$ in the horizontal jump test. The (SSBS) condition $(3.544+0.247$ second $)$ also showed significant differences $(\mathrm{p}<0.01)$ in the flying $30 \mathrm{~m}$ sprint test for faster times than the (BS-SS) condition $(3.858+0.288$ second) and also than the third condition (SPD) $(4.050+0.193$ second) . The (SPD) condition showed significant differences $(\mathrm{p}<0.01)$ in agility test for faster time than both the (BS-SS) $(11.533+0.485)$ and (SS-BS) $(11.676+0.530)$ conditions. Depending on the study results and previous studies, (SS-BS) which may be practiced before high intensity activities in trained athletes if preceded by moderate intensity running activities is recommended.
\end{abstract}

Keywords: Ballistic Stretching, Static Stretching, Kinematic Performance

\section{Introduction}

Stretching, Flexibility, and Warming-Up are all correlated, and scientific opinions vary on their relationships, proper applications and effects. Pre-exercise stretching protocols are commonly practiced, despite limited scientific evidence recommend and support one protocol over another. For this reason, warm up protocols vary according to the great variation of sport activities and tend to reflect the experience of individual trainers and athletes [1]. Traditionally, the inclusion of warming up involving static stretching exercises has been commonplace in a large number of sport activities. This stemmed from the belief that static stretching will lead to a better performance and decrease the risk of injury [2] [3] [4] [5] [6]. Some studies have reported that static stretching of short duration $(<30 \mathrm{sec})$ may actually improve acute speed performance, whereas SS of moderate duration may not obstruct both speed and agility performance related to the total duration of each performed exercise and not to the number of repetitions [7]. Although a joint is inherently capable of continued motion, the range of motion may still be limited by the resistance of the surrounding tissues which need to be stretched to their limit. This can be achieved either by applying external force (passively) or by moving a body (or a limb) (actively). When a limb moves ballistic, rapid, forceful, the achievable range may exceed that achieved by 
slow to moderate movement. Exercises that are performed rapidly by concentric contractions of the antagonist muscle group refer to the dynamic stretching (DS) training. The speed of this form of stretching ranges from slow (low momentum) to ballistic stretching (BS) (high momentum) [8]. Ballistic stretching exercises through frequent rabid swinging actions with no pause at any stage of movement force the muscle to elongate [9]. Although repeatedly rapid swinging movements lead to muscle elongation, it also activates the stretch reflex response which makes ballistic stretching usually discouraged because of the possibility of causing injury [9]. Marcos A. et al. reported that static and ballistic stretching are discouraged before a resistance training session because a pre-exercise stretching session hinders the subsequent resistance training performance according to their results[10]. Dynamic- and ballistic stretching are similar in using fast body movements to stretch the muscle, but in dynamic stretching there are no bounces or "jerky" movements. Furthermore, dynamic stretching exercises involve low intensity sport-specific movements required for the activity [11]. Although some studies declared that static stretching-induced impairments, some other studies have declared no deficits for running economy [12], sprint time[13], and jump performance [14] [15]. Nevertheless, DS or BS has been reported to increase power performance [1], [16], [17], agility [1], [6], sprint time [18] [19], and vertical jump.

The recent stretch researches include some studies that aimed to investigate the effects of different stretching techniques on the muscle tendon unit (MTU) of the gastrocnemius medialis (GM), which reported that ballistic stretching exercises of the lower-leg muscle increases dorsiflexion range of motion (ROM) but have no effect on muscle and tendon tissue. There was an increase in (ROM) due to ballistic stretching from $33.8^{\circ}$ to $37.8^{\circ}$ ( $\left.\mathrm{p}=0.00\right)$ more than static stretching from $30.9^{\circ}$ to $36.3^{\circ}(p=0.00)$ [20] [21].

N.T. Kruse et al. recommended that athletes should incorporate dynamic stretching (DS) instead of static stretching (SS) on the pre-competition warm-up which demands quickly and maximally jumps. It was reported that (DS) exercises significantly improved upon kinetic variables (rate of force development, peak force, and time-to-takeoff) compared with (SS) at 1 min after stretching. [22]

Hence, the aim of the present study was to (a) Compare the effects of combining between static stretching to the point of tension (POT) and ballistic stretching, (b) Changing of sequencing $\mathrm{BS}$ and $\mathrm{SS}$ to examine the effects on the kinematic performance Jumping, Agility and Speed. (c) Examining soccer passing drills (SPD) involving dynamic stretching on the high intensity required performances. It was hypothesized that (SS) to the (POT) followed by (BS) would result in enhanced performance compared with both (BS) followed by (SS) to the (POT) and (SPD).

\section{Methods}

The present study investigated and compared the effect of three different protocols of warming up involving stretching on the kinematic performance of horizontal jumping, Agility and speed for trained athletes. The three protocols were (a) $\mathrm{BS}+\mathrm{SS}$ to the point of tension (POT), (b) SS (POT) + BS (c) soccer passing drills (SPD) for 10 minutes without stretching. The three protocols were applied after 5 minutes of running activities. Each warming up protocol was applied two times a week and with two interval days between each time. Both, the first and second warm-up protocols differed only in the order of the mode of stretching used, whereas all other exercises used in the warm-up were identical. Performance tests of flying $30 \mathrm{~m}$ sprint, horizontal jump, and agility were conducted after each warm-up protocol. All the tests were filmed using a high speed video camera (120 frames per second) and analyzed using Kinovea 8.23 program for the kinematic analysis.

Subjects

This study was applied on 23 trained male student-athletes aged $(20+0.4$ years $)$. All testing procedures used in this present study were familiar with all subjects before the experiment began, as the tests were commonly used to evaluate their fitness status.

\section{Procedures}

Three warm-up protocols that differentiated by their order of the mode of stretching used: BS followed by SS to the point of tension (POT), SS (POT) followed by BS, and soccer passing drills (SPD) for 10 minutes without stretching. These 3 protocols were conducted on 3 separate days in that order. Each warm-up followed the same procedure, consisting of the following:

- Worming up exercises including jogging, high knee jogging, butt kicks, sidestepping and back jogging, vertical leap, and jump and jock for 5 minutes.

- Stretching protocols:

- $10 \mathrm{BS}$ exercises were performed each exercise 15 repetitions $\times 2$ sets for both sides then 10 SS exercises in each exercise the player held 15 seconds to the POT before changing immediately to the contralateral side showed in table.

Description of the stretching routines:

- Ballistic Stretching: Forward Leg Swing, Sideways Leg Swing, High knee kick exercise (variation), Bent-over Torso Twist, side lunges exercise, forward lunges exercise, Arm swings, High row, Arm Swings (chestshoulders).

- Static stretching: Standing quad stretch, groin stretchers, hurdlers stretch (Quadriceps), hurdlers stretch (hamstring), kneeling lunge stretch, overhead trunk side stretch, side lunge static, forward lunge static, Long Adductor Stretch, Triceps Stretch.

- Soccer passing drills: with variations of running with and without the ball and changing in direction

- $10 \mathrm{SS}$ exercises in each exercise the player held 15 seconds to the POT before changing immediately to the contralateral side then 10 BS exercises were performed each exercise 15 repetitions $\times 2$ sets for both sides showed in table (1). 
- SPD without stretching.

- While SS Subjects were told to stretch until they approached the end of the ROM (POT) not the point of pain within (pain threshold).

- While BS Subjects were told to perform rapid swinging and rebounding movements to force a body part past its normal range of motion ROM.
- After every stretching protocol two minutes were given as a rest before the testing session.

- All performance tests were performed outdoor in a soccer field starting with horizontal jump test then Agility test and ending with flying $30 \mathrm{~m}$ sprint test as shown in Figure 1. a, b and c.
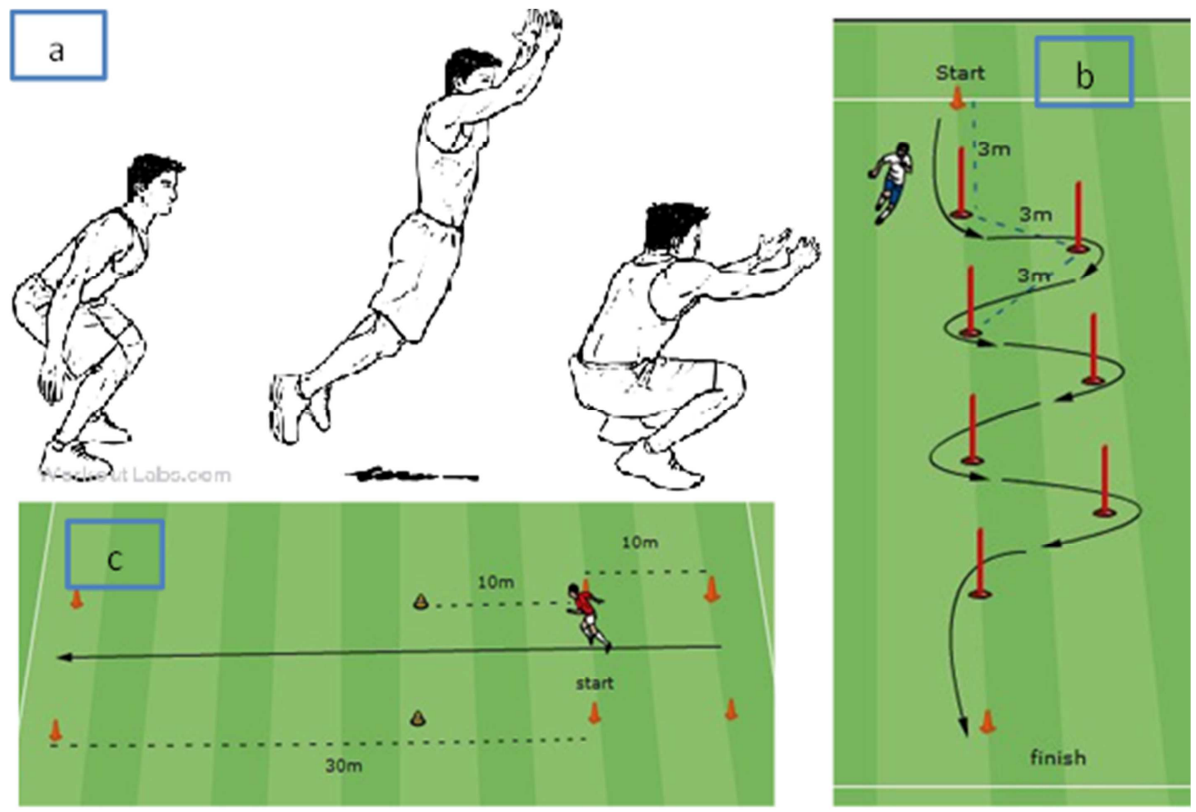

Figure 1. a) Horizontal jump test. b) Agility test. c) Fly $30 \mathrm{~m}$ sprint test.

Subjects were given two maximal attempts at each test and the best achieved time/distance was retained for statistical analysis.

Each warming up protocol was applied two times a week and with two interval days between each time. After each protocol the subjects were tested as shown in figure 2 .

The Correlation between the two measures was calculated to insure about the reliability between the test and re-test.

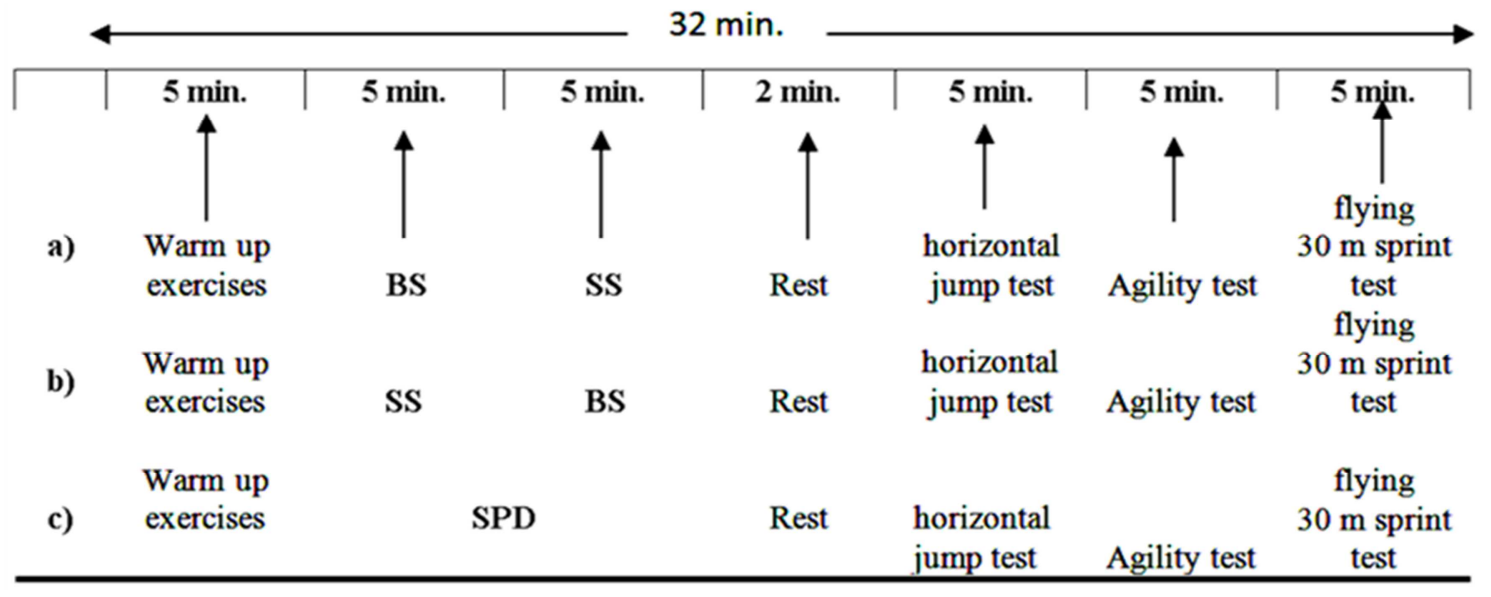

Figure 2. Timeline during each data-collection session.

\section{Statistical Analyses}

Repeated-measures analysis of variance (ANOVA) was used to compare the kinematic performances after the 3 different warm-ups. LSD post hoc analysis was used to identify pairwise differences. Statistical significance was accepted at $\mathrm{p}<0.05, \mathrm{p}<0.01$.

\section{Results}

The mean scores $( \pm \mathrm{SD})$ for the performance measures after the different warm-up protocols showed significant differences $(p<0.01)$ for The (SS-BS) condition for longer distance than the (BS-SS) condition and also than the (SPD) condition in the horizontal jump test. Horizontal jump 

Stretching on the Kinematic Performance of Horizontal Jumping, Agility and Speed for Soccer Players

kinematic variables showed significant differences $(p<0.05)$ for top flight distance for the SPD protocol. Flight angle showed significant differences $(\mathrm{p}<0.01)$ for The $(\mathrm{SS}-\mathrm{BS})$ protocol. Flight time showed no significant differences between the three warm-up protocols as shown in table (1).

Table 1. ANOVA and Pairwise Comparisons between three protocols for kinematic of horizontal jumping $n=23$.

\begin{tabular}{|c|c|c|c|c|c|c|}
\hline \multirow{2}{*}{ VAR } & \multirow{2}{*}{$X^{-} \pm$S.D } & \multicolumn{3}{|l|}{ LSD } & \multirow{2}{*}{ f } & \multirow{2}{*}{ sig } \\
\hline & & & $(S S-B S)$ & (SPD) & & \\
\hline \multirow{3}{*}{ Horizontal jumping } & $2.165 \pm 0.12$ & (BS-SS) & $-.083^{*}$ & -.033 & & \\
\hline & $2.247 \pm 0.10$ & $(S S-B S)$ & & .050 & $11.33 * *$ & .000 \\
\hline & $2.197 \pm 0.11$ & $(S P D)$ & & & & \\
\hline \multirow{3}{*}{ Top flight distance } & $1.05 \pm 0.07$ & (BS-SS) & $-.114^{*}$ & $-.127^{*}$ & & \\
\hline & $1.17 \pm 0.12$ & $(S S-B S)$ & & -.012 & $25.69 * *$ & .000 \\
\hline & $1.18 \pm 0.04$ & $(S P D)$ & & & & \\
\hline \multirow{3}{*}{ Flight angle } & $16 \pm 3$ & (BS-SS) & -.522 & $2.435 * *$ & & \\
\hline & $17 \pm 3$ & $(S S-B S)$ & & $2.957^{* *}$ & $8.02 * *$ & .003 \\
\hline & $14 \pm 2$ & $(S P D)$ & & & & \\
\hline \multirow{3}{*}{ Flight time } & $1.659 \pm 0.147$ & (BS-SS) & -.074 & -.047 & & \\
\hline & $1.733 \pm 0.129$ & $(S S-B S)$ & & .028 & 1.80 & .190 \\
\hline & $1.708 \pm 0.067$ & $(S P D)$ & & & & \\
\hline \multirow{3}{*}{ Agility } & $11.676 \pm 0.530$ & (BS-SS) & .142 & $.546^{*}$ & $18.15 * *$ & .000 \\
\hline & $11.533 \pm 0.485$ & $(S S-B S)$ & & $.403^{*}$ & & \\
\hline & $11.130 \pm 0.393$ & $(S P D)$ & & & & \\
\hline \multirow{3}{*}{ Pass Angle } & $63 \pm 3.5$ & (BS-SS) & $-1.957-^{*}$ & -1.717 & 3.05 & .069 \\
\hline & $65 \pm 2.7$ & $(S S-B S)$ & & .239 & & \\
\hline & $65 \pm 2.2$ & $(S P D)$ & & & & \\
\hline \multirow{3}{*}{$\begin{array}{l}\text { FLY 30M SPRINT } \\
\text { (sec.) }\end{array}$} & $3.858 \pm .288$ & (BS-SS) & $.314^{*}$ & $-.192^{*}$ & $26.49 * *$ & .000 \\
\hline & $3.544 \pm .247$ & $(S S-B S)$ & & $-.507^{*}$ & & \\
\hline & $4.050 \pm .193$ & $(S P D)$ & & & & \\
\hline \multirow{3}{*}{$\begin{array}{l}\text { Speed } \\
(\mathrm{m} / \mathrm{s})\end{array}$} & $7.82 \pm 0.68$ & (BS-SS) & $-.687^{*}$ & $.404^{*}$ & $23.85 * *$ & .000 \\
\hline & $8.50 \pm 0.59$ & $(S S-B S)$ & & $1.091^{*}$ & & \\
\hline & $7.42 \pm 0.36$ & $(S P D)$ & & & & \\
\hline \multirow{3}{*}{$\begin{array}{l}\text { Acceleration } \\
\left(\mathrm{m} / \mathrm{s}^{2}\right)\end{array}$} & $2.06 \pm 0.39$ & (BS-SS) & $-.367^{*}$ & $.213^{*}$ & $23.14 * *$ & .000 \\
\hline & $2.42 \pm 0.34$ & $(S S-B S)$ & & $.580^{*}$ & & \\
\hline & $1.84 \pm 0.18$ & $(S P D)$ & & & & \\
\hline \multirow{3}{*}{ STEPS in first $10 \mathrm{~m}$} & $5.7 \pm 0.38$ & (BS-SS) & $.421^{*}$ & -.221 & $8.89 * *$ & .002 \\
\hline & $5.3 \pm 0.41$ & $(S S-B S)$ & & $-.641^{*}$ & & \\
\hline & $6.0 \pm 0.66$ & $(S P D)$ & & & & \\
\hline
\end{tabular}

*The mean difference is significant at the .05 level.

** The mean difference is significant at the .01 level.

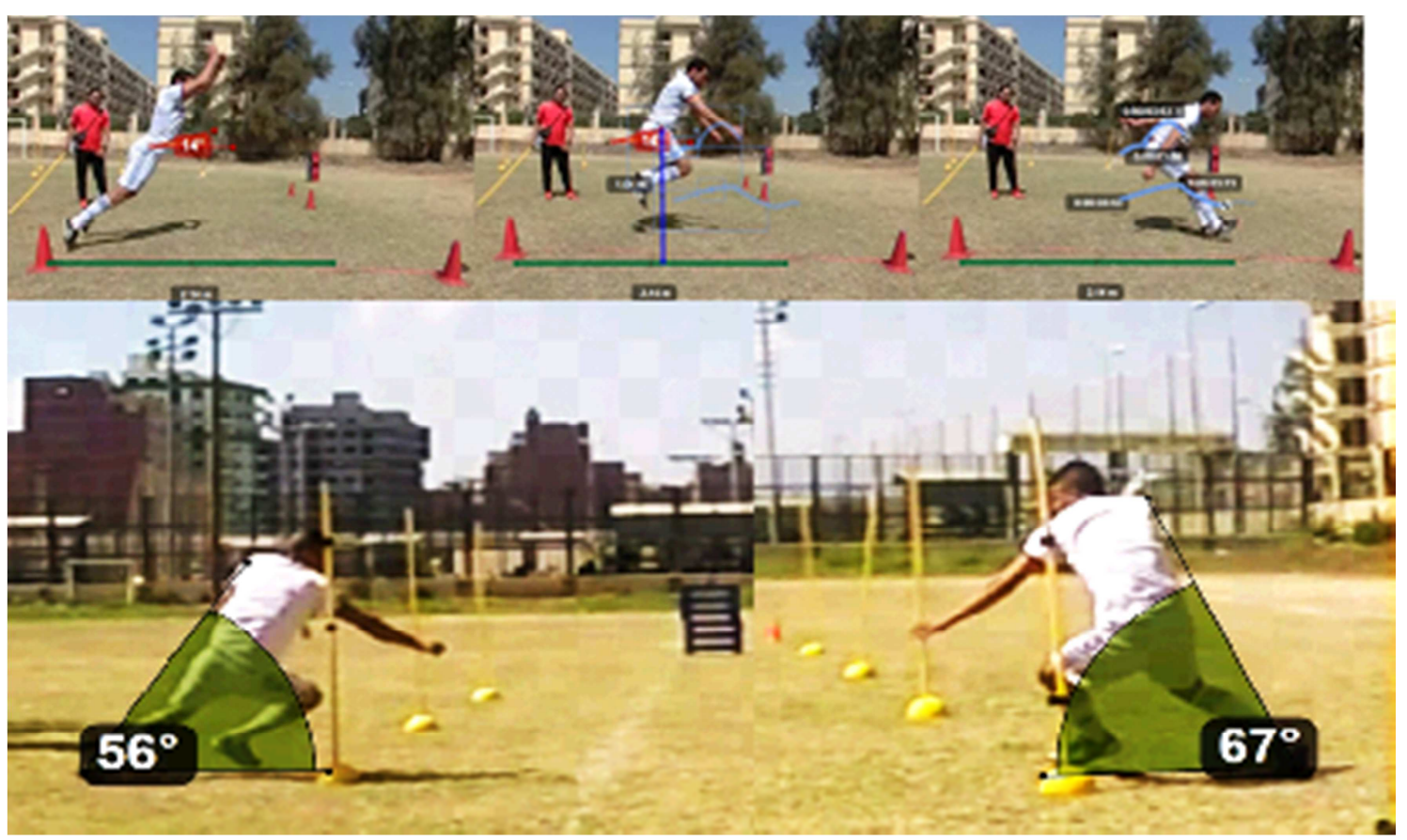

Figure 3. Kinematic analysis of horizontal jumping and agility test variables. 
The (SPD) protocol showed significant differences $(\mathrm{p}<$ $0.01)$ in agility test for faster time than both the (BS-SS) and (SS-BS) protocols. Pass angle showed non-significant differences between the three warming-up protocols, whereas there were significant differences $(\mathrm{p}<0.05)$ between $(\mathrm{BS}+\mathrm{SS})$ and $(\mathrm{SS}+\mathrm{BS})$ for the $(\mathrm{SS}+\mathrm{BS})$ as shown in table (1).

The (SS-BS) protocol showed significant differences $(\mathrm{p}<$ 0.01 ) in the flying $30-\mathrm{m}$ sprint test for faster times, speed, acceleration, and Step frequency in first $10 \mathrm{~m}$. than the (BS$\mathrm{SS})$ condition and the third condition (SPD) as shown in table (1).

\section{Discussion}

The findings of the present study were that the second warm-up protocol SS-BS positively affects the performance of the horizontal jumping and fly $30 \mathrm{~m}$ sprint whilst the SPD positively affects the agility performance. The BS-SS protocol did not affect negatively the performance however it showed better results in fly $30 \mathrm{~m}$ sprint than the SPD protocol.

Depending on the results of the present study our data supports that the SS-BS protocol has a detrimental effect on high-speed and horizontal jumping performance as this protocol depends on firstly the static stretching by placing the body into a position whereby the muscle to be stretched to the POT then secondly the rapid swinging and rebounding movements of the BS, which force the body limb to work beyond its normal range of motion. That helped the subjects reaching the optimal body angles during the takeoff and flight phase in horizontal jump test and increasing the length of the running step and decreasing the step frequency in the first $10 \mathrm{~m}$ of the flying $30 \mathrm{~m}$ sprint test. This order of the stretching protocol could be effective in high intensity activities such as Sprinting or jumping. Additionally, ballistic stretching has been reported to enhance performance in power [1], [16], [17], agility [1], [6], sprint time [18]. The SPD protocol produced better performance in Agility test than the two other protocols. It depends on the soccer specific movements which involve dynamic stretching. Practically, dynamic stretching can be achieved by performing a sport-specific warm-up but at a lower intensity. This type of warming up could be recommended before the activities that needs coordination and rapid changes in whole body position. As it benefits gaining the best pass angle to overcome the influence of gravity while rapid changing in directions. Agreed with Little and Williams that dynamic stretching is probably optimal for the speed performances required in sports such as soccer. Dynamic stretching mixed with sport specific movements is an active process, and the performance benefits obtained may stem from simplified motor control via repetitions of specific movements [6].

The BS-SS protocol produced better performance than the SPD protocol and less than the SS-BS protocol in flying $30 \mathrm{~m}$ sprint. The order of the exercises BS then SS may affected positively in the performance of 30 sprint due to firstly, lengthen of the muscle beyond the ROM while performing ballistic exercises and secondly, reaching the POT while performing SS after that as the subjects were told to hold in the POT for 15 seconds. These two points were involved in both BS-SS and SS-BS protocol in different order, whereas not in the SPD protocol as it was without stretching. After all this result disagree with [12], [23] that no sequencing effect of DS and static stretching was found in their studies as there were no statistically significant impairments associated with static stretching.

\section{Practical Applications}

Static stretching that followed by ballistic stretching during warm-ups, as opposed to no stretching or ballistic followed by static stretching, is likely most effective as preparation for the high intensity performances required in sports such as sprinting and jumping for the highly trained athletes. The inclusion of active dynamic stretching and static dynamic stretching within the warm-up routine activities can improve both flexibility and power measures as well [24]. Through the literature review and the results of previous studies, it is recommended to perform Static stretching at the end of the workout after as it helps in muscle relaxation.

\section{Conclusion}

In conclusion, static stretching followed by ballistic stretching and dynamic stretching which involves in specific skills and soccer drills should be recommended before the high intensity performances required in sports such as sprinting and jumping in soccer. Further research is needed to investigate the influence of different stretching strategies for several periods of time in different populations and of different training levels on kinematic performance and muscle architecture of both the upper and lower limps.

\section{References}

[1] D. C. T. Danny J. Mcmillian, Josef H. Moore, Brian S. Halter, "Dynamic vs. static-stretching warm up: The effect on power and agility performance," J. Strength Cond. Res. 2006, 20(3), 492-499, vol. 20, no. 3, pp. 492-499, 2006.

[2] D. G. Behm and A. Kibele, "Effects of differing intensities of static stretching on jump performance," Eur. J. Appl. Physiol., vol. 101, no. 5, pp. 587-594, 2007.

[3] S. P. Magnusson, E. B. Simonsen, P. Aagaard, P. DyhrePoulsen, M. P. McHugh, and M. Kjaer, "Mechanical and physiological responses to stretching with and without preisometric contraction in human skeletal muscle," Arch. Phys. Med. Rehabil., vol. 77, no. 4, pp. 373-378, 1996.

[4] S. P. Magnusson, E. B. Simonsen, P. Aagaard, and M. Kjaer, "Biomechanical Responses to Repeated Stretches in Human Hamstring Muscle In Vivo," Am. J. Sports Med., vol. 24, no. 5, pp. 622-628, Sep. 1996. 
[5] D. G. Behm and A. Chaouachi, "A review of the acute effects of static and dynamic stretching on performance," Eur. J. Appl. Physiol., vol. 111, no. 11, pp. 2633-2651, 2011.

[6] T. and A. G. W. Little, "Effects of differential stretching protocols during warm-ups on high-speed motor ca- pacities in professional soccer players.," J. Strength Cond. Res., vol. 20, no. 1, pp. 203-207, 2006.

[7] A. Avloniti, A. Chatzinikolaou, I. G. Fatouros, M. Protopapa, I. Athanailidis, C. Avloniti, D. Leontsini, G. Mavropalias, and A. Z. Jamurtas, "The effects of static stretching on speed and agility: One or multiple repetition protocols?," Eur. J. Sport Sci., vol. 1391, no. April, pp. 1-7, 2015.

[8] J. H. Laurence E. Holt, Thomas E. Pelham, Flexibility: A Concise Guide: To Conditioning, Performance Enhancement, Injury Prevention, and Rehabilitation (Musculoskeletal Medicine, 7 Jan. 200. Humana Press; 2008, 2008.

[9] Arnold G. Nelson and J. Kokkonen, STRETCHING ANATOMY, no. 1. Human Kinetics, 2007.

[10] M. A. Sá, G. R. Neto, P. B. Costa, T. M. Gomes, C. M. Bentes, A. F. Brown, and J. S. Novaes, "Acute effects of different stretching techniques on the number of repetitions in a single lower body resistance training session.," J. Hum. Kinet., vol. 45, pp. 177-85, Mar. 2015.

[11] B. Appleton, Stretching and Flexibility Everything you never wanted to know. Copyright c 1993-1998 by Bradford D. Appleton, 1998.

[12] P. R. Hayes and A. Walker, "Pre-exercise stretching does not impact upon running economy.," J. Strength Cond. Res., vol. 21, no. 4, pp. 1227-1232, 2007.

[13] R. E. Vetter, "Effects of six warm-up protocols on sprint and jump performance.," J. Strength Cond. Res., vol. 21, no. 3, pp. 819-23, Aug. 2007.

[14] D. P. 2 and C. K. Dimitris Chatzopoulos 1, Christos Galazoulas 1, "An Acute Bout of Static Stretching: Effects on Force and Jumping Performance," Journal of Sports Science and Medicine, 2014. [Online]. Available: file://C:/Users/user/Downloads/1 - Stretching and Performance - 2004 (1).pdf. [Accessed: 27-Jan-2016].

[15] W. Young, G. Elias, and J. Power, "Effects of static stretching volume and intensity on plantar flexor explosive force production and range of motion," J. Sports Med. Phys. Fitness, vol. 46, no. OCTOBER, pp. 403-411, 2006.

[16] K. I. 1 Taichi Yamaguchi 1, "Effects of static stretching for 30 seconds and dynamic stretching on leg extension power," $J$. Strength Cond. Res., vol. 19, no. 3, pp. 677-683, 2005.

[17] T. Yamaguchi, K. Ishii, M. Yamanaka, and K. Yasuda, “Acute effects of dynamic stretching exercise on power output during concentric dynamic constant external resistance leg extension.," J. Strength Cond. Res., vol. 21, no. 4, pp. 1238 44, 2007.

[18] A. R. Fletcher IM1, "The acute effects of combined static and dynamic stretch protocols on fifty-meter sprint performance in track-and-field athletes.," J. Strength Cond. Res., vol. 21, no. 3, pp. 784-787, 2007.

[19] M. T. Woolstenhulme, C. M. Griffiths, E. M. Woolstenhulme, and A. C. Parcell, "Ballistic stretching increases flexibility and acute vertical jump height when combined with basketball activity.," J. Strength Cond. Res., vol. 20, no. 4, pp. 799-803, 2006.

[20] A. Konrad, "EFFECTS OF DIFFERENT STRETCHING TECHNIQUES ON MUSCLE- AND TENDON," Conf. 20th Anu. Congr. Eur. Coll. Sport Sci. Malmö, no. june, 2015.

[21] A. Konrad and M. Tilp, "Effects of ballistic stretching training on the properties of human muscle and tendon structures.," $J$. Appl. Physiol., vol. 117, no. 1, pp. 29-35, Jul. 2014.

[22] N. T. Kruse, M. W. Barr, R. M. Gilders, M. R. Kushnick, and S. R. Rana, "Effect of different stretching strategies on the kinetics of vertical jumping in female volleyball athletes," $J$. Sport Heal. Sci., vol. 4, no. 4, pp. 364-370, Dec. 2015.

[23] A. Chaouachi, C. Castagna, M. Chtara, M. Brughelli, O. Turki, O. Galy, K. Chamari, and D. G. Behm, "Effect of warm-ups involving static or dynamic stretching on agility, sprinting, and jumping performance in trained individuals.," $J$. Strength Cond. Res., vol. 24, no. 8, pp. 2001-11, Aug. 2010.

[24] L. Turki-Belkhiria, A. Chaouachi, O. Turki, H. Chtourou, M. Chtara, K. Chamari, M. Amri, and D. G. Behm, "Eight weeks of dynamic stretching during warm-ups improves jump power but not repeated or single sprint performance," Eur. J. Sport Sci., vol. 14, no. February 2014, pp. 1-9, 2012. 